\title{
Uma breve revisão histórica sobre a construção dos conceitos do Autismo Infantil e da síndrome de Asperger
}

\author{
A brief historic review of the conceptions of Autism and \\ Asperger syndrome
}

\author{
Ana Carina Tamanaha ${ }^{1}$, Jacy Perissinoto ${ }^{2}$, Brasilia Maria Chiari ${ }^{3}$
}

\begin{abstract}
RESUMO
O objetivo deste estudo foi revisar historicamente os conceitos do Autismo Infantil e da síndrome de Asperger. Por meio de revisão de literatura os autores buscaram mostrar as modificações, ao longo do tempo, das concepções teóricas e das descrições clínicas destes quadros.
\end{abstract}

Descritores: Transtorno autístico; síndrome de Asperger; Linguagem; Revisão

\section{INTRODUÇÃO}

Os quadros que compõem o espectro autístico caracterizam-se pela tríade de impedimentos graves e crônicos nas áreas de interação social, comunicação verbal e não verbal e interesses. Dentre os transtornos pertencentes a esta condição, estão o Autismo Infantil e a síndrome de Asperger.

Embora existam inúmeras manifestações clínicas, qualitativamente semelhantes, é importante salientar as peculiaridades presentes em cada um dos transtornos.

O objetivo deste estudo foi revisar historicamente o construto teórico acerca dos quadros de Autismo Infantil e da síndrome de Asperger.

Trabalho realizado no Departamento de Fonoaudiologia da Universidade Federal de São Paulo - UNIFESP - São Paulo (SP), Brasil.

(1) Doutora, Professora Convidada do Departamento de Fonoaudiologia da Universidade Federal de São Paulo - UNIFESP - São Paulo (SP), Brasil; Supervisora do Laboratório de Investigação Fonoaudiológica de Linguagem - Transtornos Globais do Desenvolvimento do Departamento de Fonoaudiologia da Universidade Federal de São Paulo - UNIFESP - São Paulo (SP), Brasil.

(2) Doutora, Professora Associada do Departamento de Fonoaudiologia da Universidade Federal de São Paulo - UNIFESP - São Paulo (SP), Brasil; Coordenadora do Laboratório de Investigação Fonoaudiológica de Linguagem - Transtornos Globais do Desenvolvimento do Departamento de Fonoaudiologia da Universidade Federal de São Paulo - UNIFESP - São Paulo (SP), Brasil.

(3) Livre Docente, Professora Titular do Departamento de Fonoaudiologia da Universidade Federal de São Paulo - UNIFESP - São Paulo (SP), Brasil.

Endereço para correspondência: Ana Carina Tamanaha. R. Monsenhor Naline, 17, Parque Jabaquara, São Paulo - SP, CEP 04358-030. E-mail: anacarina.otor@unifesp.epm.br

Recebido em: 1/10/2007; Aceito em: 20/6/2008

\section{REVISÃO DE LITERATURA}

Autismo Infantil e Síndrome de Asperger: as descrições iniciais

O Autismo Infantil foi definido por Kanner ${ }^{(1)}$, em 1943, sendo inicialmente denominado Distúrbio Autístico do Contato Afetivo, como uma condição com características comportamentais bastante específicas, tais como: perturbações das relações afetivas com o meio, solidão autística extrema, inabilidade no uso da linguagem para comunicação, presença de boas potencialidades cognitivas, aspecto físico aparentemente, normal, comportamentos ritualísticos, início precoce e incidência predominante no sexo masculino.

Em 1944, Asperger ${ }^{(2)}$ propôs em seu estudo a definição de um distúrbio que ele denominou Psicopatia Autística, manifestada por transtorno severo na interação social, uso pedante da fala, desajeitamento motor e incidência apenas no sexo masculino. $\mathrm{O}$ autor utilizou a descrição de alguns casos clínicos, caracterizando a história familiar, aspectos físicos e comportamentais, desempenho nos testes de inteligência, além de enfatizar a preocupação com a abordagem educacional destes indivíduos.

Ambos os trabalhos tiveram impacto na literatura mundial; no entanto, em momentos distintos.

As descrições de Kanner ${ }^{(1)}$ foram rapidamente absorvidas pela comunidade científica. A abordagem etiológica do Autismo Infantil, proposta pelo autor, salientava a existência de uma distorção do modelo familiar, que ocasionaria alterações no desenvolvimento psico-afetivo da criança, decorrente do caráter altamente intelectual dos pais destas crianças. Apesar desta proposição, o autor não deixou de assinalar que algum fator biológico, existente na criança, poderia estar envolvido, 
uma vez que as alterações comportamentais eram verificadas precocemente, o que dificultaria a aceitação puramente relacional.

Esses referenciais para a hipótese etiológica foram, de certo modo, precursores de duas abordagens teóricas distintas para o estudo do Autismo Infantil, ainda hoje motivo de controvérsias.

A teoria afetiva propõe uma etiologia puramente relacional e, embora faça a distinção entre a síndrome descrita por Kanner e sua abordagem clínica, considera que o autismo é mais um sintoma do quadro clínico de Psicose Infantil e menos uma entidade nosológica em $\mathrm{si}^{(3-5)}$.

Ainda hoje, a teoria afetiva é preconizada e difundida na literatura psicanalítica, embora alguns autores tenham revisto seus referenciais teóricos, a respeito da etiologia, como é o caso de uma das precursoras ${ }^{(5)}$ desta teoria, que revisou os próprios conceitos teóricos acerca da definição de autismo. Segundo a autora, à luz das descobertas produzidas pela observação dos comportamentos dos bebês, não seria mais possível postular uma fase de autismo primário infantil, uma vez que, são observadas, desde muito cedo, reações do neonato ao meio. Sugeriu que o autismo deveria ser compreendido, apenas, como sendo uma condição patológica. Além disso, questionou a presença de possíveis alterações orgânicas, na criança, não detectáveis em exames disponíveis naquele momento.

Em estudo subseqüente ${ }^{(6)}$, reafirmou que a existência de uma abordagem puramente relacional para o autismo não poderia explicar a precocidade das manifestações comportamentais, relatadas pelos pais e hipotetizou a predisposição da criança como possível desencadeador da condição patológica.

Em busca de evidências que justificassem a precocidade das manifestações clínicas, os conceitos teóricos sobre a etiologia e dinâmica do Autismo se modificaram gradativamente, ao longo do tempo. Desse modo, em contrapartida à concepção original da etiologia afetiva e de incapacidade relacional, há as abordagens que buscam uma etiologia orgânica para o quadro e o caracterizam, prioritariamente, por falhas cognitivas e sociais.

Passou-se a discutir os conceitos do Autismo sob os diferentes pontos de vista teóricos. Em um dos primeiros estudos delineados, o autor buscou fazer a distinção terminológica entre o Autismo, a Esquizofrenia e a Psicose Infantil. Relacionou as diferenças existentes entre as abordagens: psicogênica e orgânica, esta última relacionada ao estudo do Sistema Nervoso Central. Além disso, discorreu sobre as bases genéticas do Autismo Infantil. Enfatizou a necessidade da avaliação das alterações da linguagem e das anormalidades perceptuais presentes nestes indivíduos. Sugeriu que muitas das manifestações podem ser explicadas pelas falhas cognitivas e de percepção ${ }^{(7)}$.

Em 1978, o mesmo autor ${ }^{(8)}$ propôs critérios diagnósticos, salientando a necessidade da observação comportamental dos indivíduos. Os critérios incluíam a perda do interesse social e da responsividade; alterações de linguagem que vão desde a ausência de fala até o uso peculiar da mesma; comportamentos bizarros, ritualísticos e compulsivos; jogo limitado e rígido; início precoce do quadro, ou seja, antes dos 30 meses de vida.
Outros autores enfatizaram o estudo dos fatores neurofisiológicos e bioquímicos. Concluíram tratar-se de uma síndrome com comportamentos específicos, manifestados precocemente, com alteração em diversas áreas do desenvolvimento, tais como: percepção, linguagem e cognição $0^{(9-13)}$.

Em pesquisa recente ${ }^{(14)}$ sugeriu-se que a causa do Autismo Infantil pode estar vinculada à alteração neuroanatômica, ou seja, sujeitos autistas podem apresentar um modelo neuroanatômico, extremamente, masculino. Este fato ocorre, provavelmente, devido às altas taxas de testosterona a que os autistas são expostos, no período pré-natal. De acordo com os autores, os cérebros de homens e mulheres são diferentes. Enquanto as mulheres demonstram alta capacidade de empatização, ou seja, são hábeis em tarefas que demandam atribuição de estados mentais, inferindo e respondendo emocionalmente às situações e às pessoas; os homens são predominantemente, sistematizantes, ou seja, respondem às demandas sociais por meio de comportamentos analíticos e sistemáticos. Deste modo, os autores defenderam a idéia de que sujeitos autistas apresentam um funcionamento cerebral essencialmente sistematizante.

Outros estudos buscaram demonstrar que a chave para o fenômeno clínico do Autismo Infantil pode estar na influência genética como base para os déficits cognitivos encontrados neste distúrbio.

Já nos estudos iniciais, observamos a menção à influência genética como provável fator etiológico ${ }^{(7)}$. Outras pesquisas também salientaram a necessidade do estudo da associação entre os achados genéticos e os prejuízos cognitivos, específicos dos sujeitos autistas ${ }^{(15-16)}$.

A hipótese de que haja uma falha cognitiva que justifique os prejuízos sociais e de comunicação dos portadores de Autismo Infantil também é abordada por diversos autores, que propuseram o estudo da Teoria da Mente como alternativa para se entender as falhas sociais dos indivíduos autistas ${ }^{(17-18)}$. Segundo os autores, nos sujeitos portadores desta condição, existe uma incapacidade na identificação, compreensão e na atribuição de sentimentos, intenções, o que ocasiona prejuízos nas relações interpessoais. A inabilidade dos autistas para estabelecer relações interpessoais eficazes justifica-se pelas ausências na detecção da intencionalidade e no compartilhamento de atenção aos objetos e eventos, com os interlocutores. Essas falhas ocasionam prejuízos na atribuição e na compreensão de estados mentais, que nem sempre estão explícitos nas situações dialógicas.

\section{DISCUSSÃO}

\section{Da aproximação diagnóstica à distinção das patologias}

A aproximação diagnóstica entre os quadros clínicos descritos por Kanner ${ }^{(1)}$ e Asperger ${ }^{(2)}$ foi realizada inicialmente, no inicio da década de $70^{(19)}$, com a proposta de identificação da Psicopatia Autística, como uma entidade nosológica e delineamento de estudo comparativo entre esta condição e o quadro de Autismo Infantil.

No entanto, é com a tradução para a língua inglesa do trabalho científico de Asperger, que este estudo teve repercussão mundial $^{(20)}$. Foi proposto o uso do termo síndrome de Asperger 
em detrimento à Psicopatia Autística e a classificação desta síndrome como pertencente ao "continuum autista", com a descrição dos prejuízos específicos nas áreas da comunicação, imaginação e socialização.

Até a década de 80, alguns autores encontravam-se reticentes quanto ao uso deste diagnóstico ${ }^{(21-22)}$. No entanto, outros passaram a defender a validação da síndrome de Asperger como entidade nosológica, demonstrando que, com a distinção dos quadros, o suporte às famílias bem como o direcionamento terapêutico, seriam mais eficazes e abrangentes ${ }^{(23-25)}$. Pelo menos dois critérios foram considerados imprescindíveis para o diagnóstico diferencial: o período de aquisição da fala e a idade de identificação do diagnóstico.

Diversos estudos salientavam que a síndrome de Asperger deveria ser considerada como pertencente ao espectro autístico, tendo uma possível etiologia de base genética ${ }^{(26-31)}$.

$\mathrm{Na}$ revisão de literatura ${ }^{(32)}$ realizada com o objetivo de validação dos Distúrbios do Espectro Autístico, por exemplo, foram selecionados 20 artigos, identificados e adequadamente investigados. Os achados destas pesquisas foram comparados em vários ítens como: prevalência, etiologia possível, sintomatologia, nível intelectual, aspectos de linguagem, história familiar e testes neuropsicológicos, entre outros. Segundo o autor, existem pelo menos três grupos que podem ser distinguidos no espectro autístico: síndrome de Asperger, Autismo de funcionamento alto e Autismo baixo funcionamento, ao se considerar o nível intelectual. Entretanto, a evidência de que essas distinções clínicas tragam inferências em relação às possíveis etiologias e tratamentos, foi apenas sugerida pelo autor. A importância da distinção entre esses grupos está na promoção de debates sobre as questões nosológicas a respeito da classificação dos Transtornos Globais do Desenvolvimento.

$\mathrm{Na}$ décima revisão da Classificação Internacional de Doenças - CID $10^{(33)}$, os Transtornos Globais do Desenvolvimento foram classificados como um grupo de alterações, caracterizadas por alterações qualitativas da interação social e modalidades de comunicação, e por um repertório de interesses e atividades restrito e estereotipado. Essas anomalias qualitativas constituem uma característica global do funcionamento do indivíduo.

De acordo com esta classificação, o Autismo Infantil foi caracterizado por um desenvolvimento anormal ou alterado, manifestado antes dos três anos, apresentando perturbação do funcionamento nas áreas: interação social, comunicação e comportamento repetitivo. A incidência populacional é em torno de 2-5 indivíduos para 10.000 e o predomínio para o sexo masculino $(4: 1)$.

O quadro de síndrome de Asperger apresenta validade nosológica incerta, caracterizada por perturbação qualitativa nas áreas de interação social e interesses. Esse distúrbio distingue-se do Autismo Infantil pelo fato de que não se verifica retardo ou alteração significativa da linguagem, bem como do desenvolvimento cognitivo.

Na revisão dos critérios diagnósticos utilizados no Manual Diagnóstico e Estatístico de Transtornos Mentais - DSM IV $\operatorname{Tr}^{(34)}$, também foi proposta a classificação tanto do Autismo Infantil, quanto da síndrome de Asperger, como subcategorias dos Transtornos Globais do Desenvolvimento. Essa secção diagnóstica caracterizou-se pelos prejuízos severos e invasivos nas áreas de interação social e repertório restrito de interesses e atividades.

A distinção entre os dois quadros centralizou-se na caracterização da área da comunicação, bastante prejudicada no Autismo. Já na síndrome de Asperger, verificou-se que o DSM IV Tr considerou não haver um prejuízo significativo nas áreas da linguagem e cognição. No entanto, há menção de que algumas dificuldades na comunicação social são verificadas, tais como: a incapacidade de reconhecer as regras convencionais da conversação que regem as interações sociais e o uso restrito de múltiplos sinais não verbais, como contato visual, expressões facial e corporal.

Assinalou-se ainda que, no quadro de Autismo Infantil, houve um aumento na prevalência sugerida pelos estudos epidemiológicos, fixando-se em, aproximadamente, 15 casos por 10.000 indivíduos. As taxas para essa alteração são quatro a cinco vezes superiores no sexo masculino.

Em relação à síndrome de Asperger, as informações sobre a prevalência continuam limitadas, mas ela parece ser mais comum no sexo masculino.

Atualizando a definição dos quadros de Autismo Infantil e síndrome de Asperger como entidades diagnósticas pertencentes ao grupo de transtornos de neurodesenvolvimento, cujos processos de socialização, comunicação e aprendizado encontram-se bastante prejudicados, outro estudo ${ }^{(35)}$ discorreu e comparou o histórico, a nosologia e as características clínicas presentes nos indivíduos portadores destas alterações. Propôs-se a hipótese de que os estudos dos prejuízos nos mecanismos biológicos fundamentais relacionados à adaptação social podem levar a emergência de fenótipos altamente heterogêneos, associados aos quadros de Transtornos Globais do Desenvolvimento.

A atuação fonoaudiológica junto aos quadros que compõem os Distúrbios do Espectro Autístico, em especial, o Autismo Infantil e a síndrome de Asperger tem se sedimentado ao longo dos anos no mundo todo ${ }^{(36-38)}$. A American Speech-LanguageHearing Association (ASHA) ${ }^{(39-40)}$ tem buscado, por exemplo, criar diretrizes, por meio do Comitê Ad Hoc em Distúrbios do Espectro Autístico (Ad Hoc Committee on Autism Spectrum Disorders), para que os fonoaudiólogos identifiquem crianças portadoras de tais condições o mais precocemente possível; estabeleçam métodos de avaliação e diagnóstico, precisos e condutas terapêuticas eficazes.

\section{COMENTÁRIOS FINAIS}

Esta breve revisão histórica nos permitiu observar a evolução do conceito do Autismo Infantil, ao longo do tempo. O mesmo ocorreu com a definição da síndrome de Asperger. A busca por critérios diagnósticos mais precisos e consistentes também se evidenciou, demonstrando que o percurso para total compreensão destes distúrbios e de suas etiologias, ainda necessita de muito desbravamento por parte dos estudiosos. Entretanto, acreditamos que o conhecimento da história dos construtos destas alterações seja fundamental para os fonoaudiólogos fortalecerem suas práticas baseadas em evidências, tanto na clínica, na assessoria, quanto na pesquisa. 


\begin{abstract}
The aim of this study was to review historically the concepts of Autism and Asperger syndrome. Through literature review, the authors evidence the changes on theoretical concepts and clinical descriptions of Autism and Asperger syndrome with time.
\end{abstract}

Keywords: Autistic disorder; Asperger syndrome; Language; Review

\section{REFERÊNCIAS}

1. Kanner L. Autistic disturbances of affective contact. Nervous Child. $1943 ; 2: 217-50$.

2. Asperger H. Die "Autistischen Psychopathen" in kindesalter. Arch Psychiatr Nervenkr. 1944;110:76-136.

3. Tustin F. Autismo e psicose infantil. Rio de Janeiro: Imago; 1975.

4. Mahler M. Psicoses infantis e outros estudos. Porto Alegre: Artes Médicas; 1983.

5. Tustin F. Revised understandings of psychogenic autism. Int J Psychoanal. 1991;72(Pt 4):585-91.

6. Tustin F. The perpetuation of an error. J Am Psychoanal Assoc. 1994;42(4):1307-10. Comment on: J Am Psychoanal Assoc. 1992;40(4):1232-5.

7. Rutter M. Concepts of autism: a review of research. J Child Psychol Psychiatry. 1968;9(1):1-25.

8. Rutter M. Diagnosis and definition of childhood autism. J Autism Child Schizophr. 1978;8(2):139-61.

9. Ornitz EM, Ritvo ER. The syndrome of autism: a critical review. Am J Psychiatry. 1976;133(6):609-21.

10. Rutter M, Schopler E. Autism and pervasive developmental disorders: concepts and diagnostic issues. J Autism Dev Disord. 1987;17(2):15986.

11. Schopler E, Mesibov GB, editors. High-functioning individuals with autism. New York: Plenum Press; c1992.

12. Bishop DV. Annotation: autism, executive functions and theory of mind: a neuropsychological perspective. J Child Psychol Psychiatry. 1993;34(3):279-93.

13. Schwartzman, JS. Neurobiologia do autismo infantil. In: Schwartzman JS, Assumpção Júnior FB. Autismo infantil. São Paulo: Memnon; 1995. p 17-78.

14. Baron-Cohen S, Knickmeyer RC, Belmonte MK. Sex differences in the brain: implications for explaining autism. Science. 2005;310(5749):81923. Comment in: Science. 2006;311(5763):952; author reply 952.

15. Bailey A, Phillips W, Rutter M. Autism: towards an integration of clinical, genetic, neuropsychological and neurobiological perspectives. J Child Psychol Psychiatry. 1996;37(1):89-126. Review.

16. Frith U. Cognitive explanations of autism. Acta Paediatr Suppl. 1996;416:63-8.

17. Happé F. Autism an introduction to psychological theory. 4th ed. London: UCL Press; 1994.

18. Baron-Cohen S. Mindblindness: an essay on autism and theory of mind. Cambridge, Mass.: MIT Press; 1997.

19. van Krevelen DA. Early infantile autism and autistic psychopathy. J Autism Child Schizophr. 1971;1(1):82-6.

20. Wing L. Asperger's syndrome: a clinical account. Psychol Med. 1981;11(1):115-29.

21. Gillberg C. Asperger's syndrome and recurrent psychosis - a case study. J Autism Dev Disord. 1985;15(4):389-97.

22. Volkmar FR, Paul R, Cohen DJ. The use of "Asperger's syndrome”. J Autism Dev Disord. 1985;15(4):437-9.

23. Wing L. Clarification on Asperger's syndrome. J Autism Dev Disord. 1986;16(4):513-5.
24. Szatmari P, Bartolucci G, Finlayson A, Krames L. A vote for Asperger's syndrome. J Autism Dev Disord. 1986;16(4):515-8.

25. Szatmari P, Bremner R, Nagy J. Asperger's syndrome: a review of clinical features. Can J Psychiatry. 1989;34(6):554-60.

26. Frith U. Asperger and his syndrome. In: Frith U, editor. Autism and Asperger syndrome. Cambridge: Cambridge University Press; 1991. p 1-36.

27. Wing L. The relationship between Asperger's syndrome and Kanner's autism. In: Frith U, editor. Autism and Asperger syndrome. Cambridge: Cambridge University Press; 1991. p. 93-121.

28. Happé FG. Annotation: psychological theories of autism: the "theory of mind" account and rival theories. J Child Psychol Psychiatry. 1994;35(2):215-29.

29. Klin A. Asperger syndrome. Child Adolesc Psychiatr Clin North Am. 1994;3(1):131-47.

30. Assumpção FB. Conceito e classificação das síndromes autistícas In: Schwartzman JS, Assumpção Júnior FB. Autismo infantil. São Paulo: Memnon; 1995. p 3-13.

31. Klin A, Volkmar FR, Sparrow SS, Cicchetti DV, Rourke BP. Validity and neuropsychological characterization of Asperger syndrome: convergence with nonverbal learning disabilities syndrome. J Child Psychol Psychiatry. 1995;36(7):1127-40.

32. Szatmari P. The validity of autistic spectrum disorders: a literature review. J Autism Dev Disord. 1992;22(4):583-600.

33. Organização Mundial da Saúde. Classificação estatística internacional de doenças e problemas relacionados à saúde: CID 10. 10a. ed. São Paulo: Edusp; 1998.

34. American Psychiatric Association. Manual diagnóstico e estatístico de transtornos mentais: DSM IV. 4a ed. Porto Alegre: Artes Médicas; 2002.

35. Klin A. Autismo e síndrome de Asperger: uma visão geral. Rev Bras Psiquiatr. 2006;28(Supl 1):S3-S11.

36. Perissinoto J, organizador. Conhecimentos essenciais para atender bem a criança com autismo. São José dos Campos: Pulso Editorial; c2003.

37. Miilher LP, Fernandes FDM. Análise das funções comunicativas expressas por terapeutas e pacientes do espectro autístico. Pró-Fono. 2006;18(3):239-48.

38. Tamanaha AC. A eficácia da intervenção terapêutica fonoaudiológica nos distúrbios do espectro autístico [tese]. São Paulo: Universidade Federal de São Paulo; 2008.

39. American Speech-Language-Hearing Association [Internet]. Roles and responsabilities of speech-language pathologists in diagnosis, assessment, and treatment of autism spectrum disorders across the life span [position statement]. Rockville: ASHA; c1997-2008. [cited 2008 Jul 12]. Available from: http://www.asha.org/docs/pdf/PS2006-00105. pdf

40. American Speech-Language-Hearing Association [Internet]. Guidelines of speech-language pathologists in diagnosis, assessment, and treatment of autism spectrum disorders across the life span [guidelines]. Rockville: ASHA; c1997-2008. [cited 2008 Jul 12]. Available from: http://www. asha.org/docs/pdf/GL2006-00049.pdf. 